whites and economically active age group), except for the treatment result (the cure rate for coinfected patients is predominant, Which does not reflect the country profile, however, the death rate remains an alarming situation, as well as the hospitalisation rate for associated complications).

Conclusion The interaction of diseases requires the need for coordinated work developed by TB and HIV/AIDS programs to reduce the burden of both diseases and promote more favourable outcomes.

\section{P3.81 LATIN AMERICA PROFICIENCY TESTING PROGRAM FOR DIAGNOSIS AND ANTIMICROBIAL SUSCEPTIBILITY OF NEISSERIA GONORRHOEAE}

${ }^{1} \mathrm{P}$ Galarza, ${ }^{1} \mathrm{R}$ Gianecini, ${ }^{1} \mathrm{C}$ Oviedo, ${ }^{1} \mathrm{~K}$ Silva, ${ }^{2} \mathrm{P}$ Ramon Pardo, ${ }^{2} \mathrm{M}$ Ghidinelli, ${ }^{2} \mathrm{M}$ Galas and ${ }^{3}$ NGQCP Participants. ${ }^{1}$ Instituto Nacional de Enfermedades Infecciosas (INEI) - ANLIS. Ciudad Autonoma de Buenos Aires. Argentina; ${ }^{2}$ Pan-American Health Organisation (PAHO). World Health Organisation (WHO); ${ }^{3}$ Latin American Quality Control Comparison Program in Diagnostic and Antimicrobial Susceptibility Testing for Neisseria gonorrhoeae

\subsection{6/sextrans-2017-053264.316}

Introduction Since 2013 an ongoing international inter-laboratory quality control program (NGQCP) has analysed the capability of 16 national reference laboratories to diagnose and perform antimicrobial susceptibility testing (AST) for Neisseria gonorrhoeae in Latin America. The program is supported by PAHO/WHO for the GASP-RELAVRA network and coordinated by the Sexually Transmitted Disease Reference Laboratory (LC) - INEI from Argentina. Results of the 2016 fourth round of the NGQCP are presented.

Methods A panel with 5 isolates belong to Neisseriaceae family, is envoyed once by year. A questionnaire is attached to collect information from each codified laboratory (lab). The 2008 WHO panel and ATCC 49226 reference strains were distributed. NGQCP evaluate: presumptive and confirmatory identification and, AST by disk diffusion and Minimum inhibitory concentrations (MICs) determination by either agar dilution or Etest methods. MIC interpretations were based on the criteria of the Clinical Laboratory Standards Institute (CLSI).

Results Fifteen labs recovered isolates. Complete conventional presumptive and confirmatory identification was made by $73 \%$ and $87 \%$ of labs, respectively. Only 3 labs introduced a different confirmatory methodology. The $97.3 \%$ (72/74) of diagnostics were included in the category genus and species correct. Three (3/15) labs not realised $\beta$-lactamase detection. Disk diffusion assessment showed 31 minor, 2 major and 3 very major discrepancies. The overall agreement of MIC results (MICs $\pm 1 \log 2$ dilutions) between 8 labs, including the LC, compared to the modal MICs was 90\% (277/307). Percentage agreements by antibiotics was: penicillin 83\% (49/59); tetracycline 89\% (64/72); ciprofloxacin 93\% (53/57); ceftriaxone $90 \%$ (53/59); cefixime $88 \%(7 / 8)$ and azithromycin 98\% (51/ 52)

Conclusion NGQCP is an essential pillar for an effective surveillance program in order to ensure that their data are reliable. We expect more labs in the Region realise MIC determination by agar dilution methods and improve level of concordance for AST.

\section{P3.82 AN UPWARD TREND IN OCULAR SYPHILIS CASES IN BRITISH COLUMBIA, CANADA, 2013-2016: A DESCRIPTIVE ANALYSIS}

${ }^{1} \mathrm{G}$ Shumilak, ${ }^{2,3}$ J Wong, ${ }^{2} \mathrm{~B}$ Arnold, ${ }^{2,3} \mathrm{M}$ Gilbert, ${ }^{2} \mathrm{C}$ Lukac, ${ }^{2} \mathrm{~S}$ Makaroff, ${ }^{2} \mathrm{C}$ Montgomery, ${ }^{2,4}{ }^{1} \mathrm{D}$ Moore, ${ }^{2} \mathrm{C}$ Prescott, ${ }^{1,2} \mathrm{~T}$ Grennan. ${ }^{1}$ Division of Infectious Diseases, University of British Columbia, Vancouver, Canada; ${ }^{2} B C$ Centre for Disease Control, Vancouver, Canada; ${ }^{3}$ School of Population and Public Health, University of British Columbia, Vancouver, Canada; ${ }^{4} B C$ Centre for Excellence in HIVIAIDS, Vancouver, Canada

\subsection{6/sextrans-2017-053264.317}

Introduction HIV-positive individuals are generally considered higher risk for early and more serious neurologic complications related to syphilis. In 2014-2015, clusters of ocular syphilis cases were reported in the US. Simultaneously, the Canadian province of British Columbia (BC) saw a dramatic rise in infectious syphilis cases by $40 \%$. Here, we describe ocular syphilis cases diagnosed in BC.

Methods All neurosyphilis cases diagnosed in BC since 2013 were reviewed to identify ocular cases. Ocular syphilis was defined as having signs/symptoms of ocular disease (e.g. uveitis, blurred vision) and syphilis of any stage, as defined by the Centres for Disease Control and Prevention.

Results Between January $1^{\text {st }}, 2013$ and October 31 $1^{\text {st }}, 2016,35$ cases of ocular syphilis were recorded in BC. Most were male $(32 / 35 ; 91.4 \%)$ and identified as white $(20 / 35 ; 57.1 \%)$. The mean age was 49.7 years. A majority $(18 / 35$; 51.4\%) were living with HIV. The most frequent ophthalmologic diagnoses were uveitis (41.9\%), optic neuritis (12.9\%), and retinitis (9.7\%). Twenty-three cases had lumbar puncture data available: $13(56.5 \%)$ had elevated cerebrospinal fluid (CSF) protein, 15 (65.2\%) had elevated CSF cell count, and 6 (26.1\%) had positive CSF VDRL. As a proportion of all syphilis cases, ocular syphilis accounted for $0.80 \%$ of all cases during the 2013-2015 period, versus $1.54 \%$ for $2016(p=0.05)$. Stratified by HIV serostatus, there was a significant increase in the proportion of ocular syphilis cases in those living with HIV between the 2013-2015 and 2016 time periods $(1.17 \%$ vs. $3.21 \%, \mathrm{p}=0.03)$.

Conclusion Paralleling trends observed in some US jurisdictions, BC is experiencing an increase in ocular syphilis cases, and an increasing proportion of syphilis cases in those living with HIV are being diagnosed with ocular findings. These results further highlight the importance of continuing efforts to respond to the syphilis epidemic, and focused screening for ocular symptoms, particularly in those at highest risk.

\section{P3.83 ANTIMICROBIAL RESISTANCE OF UREAPLASMA UREALYTICUM AND MYCOPLASMA HOMINIS RESEARCHED AT A REFERENCE CENTRE IN SALVADOR, BAHIA}

${ }^{1} \mathrm{GC}$ Souza, ${ }^{2}$ E Xavier-Souza, ${ }^{2} \mathrm{MS}$ Timbó, ${ }^{1} \mathrm{~V}$ Cunha, ${ }^{1,3} \mathrm{AG}$ Travassos. ${ }^{1}$ Universidade do Estado da Bahia, Brazil; '2Universidade Federal da Bahia, Brazil; ${ }^{3}$ Centro Estadual Especializado em Diagnóstico, Assistência e Pesquisa (CEDAP), Brazil

\subsection{6/sextrans-2017-053264.318}

Introduction: Ureaplasma urealyticum (UU) and Mycoplasma hominis $(\mathrm{MH})$ are potentially pathogenic organisms commonly found in the urogenital tract, with colonisation rates up to $80 \%$ and $40 \%$ worldwide, respectively. The aim of this study was to estimate antimicrobial resistance by $\mathrm{UU}$ and $\mathrm{MH}$, and 
identify the antimicrobial agents involved in bacterial resistance.

Methods A cross-sectional study, with data obtained retrospectively through medical records review. We analysed 140 patients whose women's endocervical samples and men's urine tested positive for $\mathrm{MH}$ and UU. The test used for the diagnosis and evaluation of antimicrobial resistance was the MYCOFAST Screening EvolutioN 3 kit.

Results The majority of patients were female (90.7\%), 57.9\% of the patients had $>29$ years of age and $84.3 \%$ did not have a stable union. About $70.0 \%$ of the patients tested positive for UU, $3.0 \%$ for $\mathrm{MH}$, and $27.0 \%$ for both. HIV coinfection was seen in $32.1 \%$ and HPV in $30.0 \%$. Absence of current history of Chlamydia trachomatis was a protective factor for the acquisition of azithromycin resistance $(p=0.04)$. As for the antimicrobials, doxycycline showed sensitivity rates higher than $96 \%$ for both infections, while azithromycin showed $86.8 \%$ of sensitivity for UU, but resistance of $75 \%$ for $\mathrm{MH}$. Ciprofloxacin showed sensitivity rates lower than $15 \%$ for both infections, while less than 35\% were strains sensible to ofloxacin. Erythromycin resistance rates ranged over $65 \%$ for the UU-MH coinfection, while over $90 \%$ of sample was sensible to tetracycline and clarithromycin resistance rates ranged from $7.1 \%$ for UU to $100 \%$ for $\mathrm{MH}$.

Conclusion The use of ciprofloxacin and ofloxacin is highly debatable considering the high rates of total and intermediate resistance. In our population, doxycycline showed high efficiency and is therefore recommended for the treatment of UU and $\mathrm{MH}$ infections. Monitoring antimicrobial resistance is fundamental for the adequacy of the therapeutic recommendations.

\section{P3.84 ESTIMATING THE ANTIBODY PREVALENCE OF HERPES SIMPLEX VIRUS TYPE 1 AMONG SELECT MIDDLE EAST AND NORTH AFRICA POPULATIONS}

'Gheyath Nasrallah, ${ }^{2}$ Soha Dargham, 'Layla Mohammed, '2Laith Abu-Raddad. 'Qatar University, Doha, Qatar; ${ }^{2}$ Weill Cornell Medicine-Qatar, Doha, Qatar

\section{$10.1136 /$ sextrans-2017-053264.319}

Introduction Evidence indicates a growing role for herpes simplex virus type-1 (HSV-1) as a sexually transmitted infection and as the etiological agent of genital herpes, but HSV-1 epidemiology in the Middle East and North Africa (MENA) remains poorly understood. We aimed to measure HSV-1 antibody prevalence among select MENA populations and to characterise the infection's age-distribution.

Methods Evidence indicates a growing role for herpes simplex virus type-1 (HSV-1) as a sexually transmitted infection and as the etiological agent of genital herpes, but HSV-1 epidemiology in the Middle East and North Africa (MENA) remains poorly understood. We aimed to measure HSV-1 antibody prevalence among select MENA populations and to characterise the infection's age-distribution.

Results Country-specific HSV-1 prevalence was estimated for 10 MENA national populations of male blood donors residing in Qatar. HSV-1 prevalence was estimated at 97.5\% $(95 \%$ confidence interval (CI) 95.3\%-98.7\%) among Egyptians, 92.6\% (95\% CI 87.2\%-95.8\%) among Yemenis, 90.7\% (95\% CI 84.5\%-94.6\%) among Sudanese, 88.5\% (95\% CI 83.9\%92.6\%) among Syrians, 86.5\% (95\% CI 81.0\%-90.5\%) among
Jordanians, $82.3 \%$ (95\% CI 78.2\%-85.7\%) among Qataris, $81.4 \%$ (95\% CI 73.3\%-87.5\%) among Iranians, 81.4\% (95\% CI 73.4\%-87.4\%) among Lebanese, $80.5 \%$ (95\% CI $74.2 \%$ $85.2 \%$ ) among Palestinians, and $77.0 \%$ (95\% CI $70.7 \%$ 82.3\%) among Pakistanis. Age-specific HSV-1 prevalence was estimated among male blood donors from Egypt, the Fertile Crescent (Iraq, Jordan, Lebanon, Palestine and Syria) and Qatar. There was a trend of growing HSV-1 prevalence with age among the Fertile Crescent and Qatari nationals. HSV-1 prevalence increased from 70.0\% (95\% CI 56.3\%-80.9\%) for those aged $<24$ years up to $98.0 \%$ (95\% CI $89.5 \%-99.7 \%$ ) for those aged $\geq 55$ years in the Fertile Crescent. Similar pattern was observed for Qatar, but for Egypt, prevalence was steadily above $90 \%$ for all age groups. Our results showed no significant association between sex and HSV-1 seropositivity.

Conclusion HSV-1 prevalence in MENA continues at high level, but for the majority of nationalities, at considerably lower levels than historical levels. The decline in prevalence is most pronounced among youth. As much as a third of those $<30$ years of age are reaching sexual debut with no protective antibodies against HSV-1 genital acquisition, and accordingly, are at risk of genital herpes.

\section{P3.85 EVALUATION OF GENTAMICIN SUSCEPTIBILITY AND RESISTANCE PHENOTYPES OF NEISSERIA GONORRHOEAE ISOLATES IN ARGENTINA}

${ }^{1} \mathrm{R}$ Gianecini, ${ }^{1} \mathrm{C}$ Oviedo, ${ }^{2} \mathrm{GASSP}-\mathrm{AR},{ }^{1}$ Galarza P. ${ }^{1}$ Servicio de Enfermedades de Transmisión Sexual, Instituto Nacional de Enfermedades Infecciosas (INEI)-ANLIS "Dr. Carlos G. Malbrán", Ciudad Autónoma de Buenos Aires, Argentina.; 'Gonococcal Antimicrobial Susceptibility Surveillance Programme-Argentina (GASSP-AR)

\subsection{6/sextrans-2017-053264.320}

Introduction The emergence of multidrug resistance in N. gonorrhoeae highlights the need to consider alternatives for future therapeutic use. Nowadays, the aminoglycoside gentamicin is considered a possible treatment option for gonorrhoea, particularly in dual therapy. Gentamicin has been used successfully for several years in the treatment of gonococcal urethritis in Malawi. However, treatment failures have been reported. In Argentina, N. gonorrhoeae isolates with multiple resistance profiles to different antimicrobials, including cefixime and ceftriaxone, have been detected. Therefore, the knowledge of the susceptibility to new antimicrobial options for gonorrhoea treatment is required. The aim of this study was to determine the susceptibility to gentamicin of $N$. gonorrhoeae isolates with different resistance profiles in 2013 and 2015.

Methods We studied 237 N. gonorrhoeae isolates obtained in 2013 and 2015 from the GASSP-AR. The MICs to penicillin, tetracycline, ciprofloxacin, cefixime, ceftriaxone, azithromycin and gentamicin were determined by the agar dilution test according to CLSI guidelines.

Results Gentamicin MICs ranged from 2 to $16 \mu \mathrm{g} / \mathrm{ml}$. The $\mathrm{MIC}_{50}$ and $\mathrm{MIC}_{90}$ were $8 \mu \mathrm{g} / \mathrm{ml}$. The rate of isolates with MIC $8 \mu \mathrm{g} / \mathrm{ml}$ was $67.1 \%$, while $30.4 \%$ showed a MIC of $4 \mu \mathrm{g} / \mathrm{ml}$. A $75.5 \%$ of the isolates included in this study showed resistance to one or more of the following antibiotics: penicillin (40.5\%), tetracycline (30.4\%), ciprofloxacin $(59.9 \%)$, and $5.5 \%$ of the isolates showed decreased susceptibility to cefixime and ceftriaxone. A total of 15 resistance phenotypes 